\title{
ON THE ASYMPTOTIC EXPANSIONS OF ENTIRE FUNCTIONS DEFINED BY MACLAURIN SERIES
}

H. K. HUGHES

1. Introduction. Let $f(z)$ be an entire function defined by a Maclaurin series of the form

$$
\sum_{n=0}^{\infty} g(n) z^{n}
$$

with infinite radius of convergence. We wish, in this paper, to make some observations of a general nature relative to the asymptotic behavior of this function in the neighborhood of the point at infinity. Some restrictions will, of course, be placed on the coefficient $g(n)$ of $z^{n}$.

To begin with, we shall suppose $g(n)$ to be of such character that we can replace $n$ by the complex variable $w=x+i y$. Then we shall assume that the resulting function $g(w)$ satisfies the following two conditions when it is considered throughout any arbitrary right halfplane $x>x_{0}$ :

(a) it is single-valued and analytic;

(b) corresponding to any positive number $\epsilon$, there exists a positive constant $K$, dependent only on $\epsilon$ and $x_{0}$, such that

$$
|g(x+i y)|<K e^{(\gamma \pi+6)|y|}
$$

where $\gamma$ is a non-negative constant.

Under these conditions, if $k$ is a positive integer such that $k \geqq \gamma$, then it is known that we may express $f(z)$ in the form

$$
\begin{aligned}
f(z)= & \int_{-l-1 / 2}^{\infty} g(x)\left[(-1)^{k+1} z\right]^{x} \frac{\sin k \pi x}{\sin \pi x} d x \\
& -\sum_{n=1}^{l} g(-n) z^{-n}+\xi(z, l),
\end{aligned}
$$

where $l$ is any positive integer, and where if $\left|\arg \left[(-1)^{k+1} z\right]\right|<\pi$, we have $\lim _{|z| \rightarrow \infty} z^{l} \xi(z, l)=0$.

This theorem was first proved for the special case in which $k=1$ by W. B. Ford $\left[1\right.$, p. 30]. ${ }^{1}$ The general form (3) is due to C. V.Newsom [4]. Both Ford and Newsom have used their results in finding the asymptotic expansions of several special types of functions [1, chap-

Presented to the Society, April 28, 1944; received by the editors January 7, 1944.

1 Numbers in square brackets refer to references at the end of the paper. 
ters 6,$7 ; 5 ; 6]$. The theorem was also used in $[2]$. A study of the examples appearing in these investigations has suggested to the writer the generalities which are noted in the following sections.

2. A fundamental lemma. It will be observed that equation (3) does not furnish complete information regarding the asymptotic expansions of the function $f(z)$, due to the presence of the integral in the right-hand member. By finding the asymptotic expansions of this integral under suitable restrictions on $g(w)$, we shall arrive at a general theorem concerning such expansions of $f(z)$.

The proof of the theorem rests upon a lemma regarding the asymptotic character of a certain definite integral. This lemma is as follows:

LEMMA. Consider the function

$$
F(z)=\int_{-\lambda}^{\infty} H(x) z^{x} d x
$$

where $\lambda$ is a suffuciently large positive number. ${ }^{2}$ Suppose that the function $H(w)$, where $w=x+i y$, is single-valued and analytic in the finite w-plane and, when considered throughout the arbitrary right half-plane $x>x_{0}$, can be expressed in the form

$$
H(w)=\frac{c_{0}}{\Gamma(w+1)}+\frac{c_{1}}{\Gamma(w+2)}+\cdots+\frac{c_{s}+\delta(w, s)}{\Gamma(w+s+1)},
$$

where the c's are constants, and $\lim _{|w| \rightarrow \infty} \delta(w, s)=0 ; s=0,1,2, \cdots$. Then for values of $z$ of large modulus such that $|\arg z|<\pi$, we have

$$
F(z) \sim \begin{cases}e^{z} \sum_{n=0}^{\infty} c_{n} z^{-n} ; & |\arg z| \leqq \pi / 2, \\ 0 ; & |\arg z|>\pi / 2 .\end{cases}
$$

The proof of this lemma is omitted here since it requires only slight changes in one of the discussions appearing in the work of Ford [1, art. 20].

3. A general theorem. We proceed now to study the function $f(z)$ defined by the series (1). We shall establish the following general theorem regarding its asymptotic expansions.

ThEOREM. Given the entire function

2 The result also holds if $\lambda$ is a complex number whose real part is positive and sufficiently large. The integration then takes place from $-\lambda$ to $+\infty$ along the line parallel to the real axis. 


$$
f(z)=\sum_{n=0}^{\infty} g(n) z^{n}
$$

suppose that the function $g(w)$, where $w=x+i y$, is single-valued and analytic in the finite w-plane and, when considered throughout the arbitrary right half-plane $x>x_{0}$, can be expressed in the form

$$
g(w)=\sigma^{w}\left\{\frac{c_{0}}{\Gamma(\alpha w+t)}+\frac{c_{1}}{\Gamma(\alpha w+t+1)}+\cdots+\frac{c_{s}+\delta(\alpha w, s)}{\Gamma(\alpha w+t+s)}\right\},
$$

where $\sigma$ and $\alpha$ are positive, the c's and t are constants, and $\lim _{|w| \rightarrow \infty} \delta(\alpha w, s)$ $=0 ; s=0,1,2, \cdots$. Then, when considered for values of $z$ of large modulus such that $-\pi<\arg z \leqq \pi$, the function $f(z)$ is developable asymptotically in the form

$$
f(z) \sim \frac{1}{\alpha} \sum_{\mu}\left\{Y_{\mu}^{(1-t)} e^{Y_{\mu}} \sum_{n=0}^{\infty} c_{n} Y_{\mu}^{-n}\right\}-\sum_{n=1}^{\infty} g(-n) z^{-n},
$$

where $Y_{\mu}$ denotes the expression $\sigma^{1 / \alpha} z^{1 / \alpha} e^{2 \pi i \mu / \alpha}$, and the symbol $\sum_{\mu}$ denotes summation over those integral values of $\mu$ which satisfy $|\arg z+2 \pi \mu|$ $\leqq \pi \alpha / 2$.

Proof. Under the conditions postulated, when $x>x_{0}$, we may write

$$
|g(x+i y)|<K e^{(x \alpha / 2+e)|y|}
$$

where $\epsilon$ and $K$ have the meanings described in $\$ 1 .^{3}$ Consequently, the theorem of Ford and Newsom is applicable to the function $f(z)$ if $k$ is properly chosen. If a proper choice be made, then, for the corresponding range of arg $z$, we have

$$
f(z)=\phi(z)-\sum_{n=1}^{l} g(-n) z^{-n}+\xi(z, l),
$$

where $\phi(z)$ denotes the integral

$$
\int_{-l-1 / 2}^{\infty} g(x)\left[(-1)^{k+1} z\right] x \frac{\sin k \pi x}{\sin \pi x} d x .
$$

In particular, if $k$ is selected as the smallest odd integer $2 p+1$ such that $k \geqq \alpha / 2$, then the factor $\left[(-1)^{k+1} z\right]^{x}$ in the integrand of $\phi(z)$ becomes $z^{x}$, and (9) is then valid when $|\arg z|<\pi$. On the other hand, if $k$ is taken as $2 p+2$, then this factor becomes $(-z)^{x}$, and (9) holds for $0<\arg z=\pi+\arg (-z)<2 \pi$, and hence in particular when $\arg z=\pi$.

\footnotetext{
${ }^{8}$ For the special property of the gamma function from which this inequality results, see $[1$, p. 61$]$.
} 
Now we have

$$
\frac{\sin k \pi x}{\sin \pi x}=\left\{\begin{array}{cl}
\sum_{\mu=-p}^{p} e^{2 \pi i \mu x} ; & k=2 p+1, \\
e^{\pi i x} \sum_{\mu=-p-1}^{p} e^{2 \pi i \mu x} ; & k=2 p+2 .
\end{array}\right.
$$

And since $e^{\pi i x} \cdot(-z)^{x}=z^{x}$, it follows that for either of the two values of $k$ mentioned, the integral $\phi(z)$ is equal to the sum of $k$ integrals of the form

$$
h_{\mu}(z)=\int_{-l-1 / 2}^{\infty} g(x)\left(z \cdot e^{2 \pi i \mu}\right)^{x} d x,
$$

where we now have $-\pi<\arg z \leqq \pi$, and the integer $\mu$ takes on the values indicated in the above identities.

We shall next obtain the asymptotic expansions of the integral in (10). First, we recall that $g(x)$ is expressible in form (7). Hence, if we change the variable of integration in (10) from $x$ to $x^{\prime}$ through the transformation $\alpha x+t=x^{\prime}+1$, and then drop the primes, (10) assumes the form

$$
h_{\mu}(z)=\frac{1}{\alpha} Y_{\mu}^{(1-t)} \int_{-\lambda}^{\infty} H(x) Y_{\mu}^{x} d x
$$

where $Y_{\mu}$ is as already defined, $\lambda=\alpha(l+1 / 2)+1-t$, and $H(x)$ is of the form

$$
\frac{c_{0}}{\Gamma(x+1)}+\frac{c_{1}}{\Gamma(x+2)}+\cdots+\frac{c_{s}+\delta(x, s)}{\Gamma(x+s+1)} .
$$

We may therefore apply the fundamental lemma of $\$ 2$ to the integral appearing in (11), assuming that $l$ has been chosen sufficiently large. In (5), $z$ is to be replaced by $Y_{\mu}$, and $\arg z$ by $(\arg z+2 \pi \mu) / \alpha$. Thus, for values of $z$ such that $-\pi<\arg z \leqq \pi$ and $|\arg z+2 \pi \mu|<\pi \alpha$, we have

$$
h_{\mu}(z) \sim\left\{\begin{array}{l}
\frac{1}{\alpha} Y_{\mu}^{(1-t)} e^{Y_{\mu}} \sum_{n=0}^{\infty} c_{n}\left(Y_{\mu}\right)^{-n} ; \\
0
\end{array}\right.
$$

the first result holding when $|\arg z+2 \pi \mu| \leqq \pi \alpha / 2$, and the second otherwise.

Having established (12), we have only to sum the expansions of 
$h_{\mu}(z)$ over the proper values of $\mu$ in order to arrive at the expansion of the integral $\phi(z)$. Evidently this will yield the expansion

$$
\phi(z) \sim \frac{1}{\alpha} \sum_{\mu}\left(Y_{\mu}^{(1-t)} e^{Y_{\mu}} \sum_{n=0}^{\infty} c_{n} Y_{\mu}^{-n}\right)
$$

where $\sum_{\mu}$ has the meaning already stated.

Finally, if we replace $\phi(z)$ in (9) by its asymptotic expansion given in (13), and write the result in asymptotic notation, we arrive at (8). This concludes the proof of the theorem.

4. Special cases. Dominant terms. We shall now note the simplified forms assumed by (8) for certain values of $\alpha$ and of arg $z$. At the same time, we shall pick out the dominant terms in each form. First, if $\alpha$ is such that $0<\alpha<2$, then the condition $|\arg z+2 \pi \mu| \leqq \pi \alpha / 2$ is satisfied only by $\mu=0$ when $|\arg z| \leqq \pi \alpha / 2$. Hence for such $\alpha$ and $\arg z$, we have

$$
f(z) \sim \frac{1}{\alpha}(\sigma z)^{(1-t) / \alpha} \exp (\sigma z)^{1 / \alpha} \sum_{n=0}^{\infty} c_{n}(\sigma z)^{-n / \alpha}-\sum_{n=1}^{\infty} g(-n) z^{-n} .
$$

Here we see that the first series is dominant when $\arg z$ is confined to the narrower range $|\arg z|<\pi \alpha / 2$, but not when $|\arg z|=\pi \alpha / 2$. Furthermore, if $\alpha=2$, and $|\arg z|<\pi$, expansion (14) still holds, and the first series is dominant.

On the other hand, if we have $0<\alpha<2$ and $|\arg z|>\pi \alpha / 2$, but still such that $-\pi<\arg z \leqq \pi$, then no integral value of $\mu$ satisfies $|\arg z+2 \pi \mu| \leqq \pi \alpha / 2$. Hence, for such values of $z$, (8) reduces to the form

$$
f(z) \sim-\sum_{n=1}^{\infty} g(-n) z^{-n}
$$

It is to be noted that this result is consistent with another theorem due to Ford $[1$, p. 4] according to which (15) holds throughout the open sector $\pi \alpha / 2<\arg z<(2-\alpha / 2) \pi$.

Finally, we note that when $\alpha \geqq 2$, the terms affected by $\sum_{\mu}$ are dominant over the last series in (8), this being true for all values of $\arg z$ under consideration.

5. Supplementary remarks. Several supplementary observations relative to the theorem established in $\$ 3$ are now to be noted. In the first place, we have required that the function $g(w)$ have no finite singularities. Suppose, however, that it does have a singularity at $w=w_{0}$, while $(7)$ continues to hold for all values of $|w|$ sufficiently 
large. Then if $w_{0}$ is not a negative integer, the theorem still holds provided that we subtract from the right member of (8) the asymptotic expansion of the loop integral

$$
\frac{1}{2 i} \int_{C} \frac{g(w)\left[(-1)^{k+1} z\right]^{w}}{e^{k i \pi w} \sin \pi w} d w
$$

The loop $C$ surrounds the point $w_{0}$ and extends to infinity in any convenient direction lying in either the third or the fourth quadrant. If $w_{0}$ is a negative integer, say $-m$, then the term $g(-m) z^{-m}$ in (8) is suppressed, and the expansion of (16) is put in its place. For justification of these statements, the reader is referred to [3], where a method for finding the expansion of (16) is also discussed.

Secondly, the theorem which we have here established narrows down the difficulties involved in determining the asymptotic expansion of a given entire function of form (1). For the problem of finding the expansion of the integral appearing in (3) is solved if it can be shown that the function $g(w)$ can be written in the form (7).

Thirdly, the theorem can evidently be applied to a large variety of entire functions. In many instances in which the coefficient $g(n)$ involves the gamma function, relation (7) is satisfied. In this connection, we note that if the value of $\sigma$ in (7) is unity, then $f(z)$ is necessarily the function

$$
F_{\alpha}(z, t)=\sum_{n=0}^{\infty} \frac{h(n)}{\Gamma(\alpha n+t)} z^{n}
$$

which was discussed in [2]. The writer plans to apply the theorem to certain further important types of functions, and to report the results in later papers.

\section{REFERENCES}

1. W. B. Ford, The asymptotic developments of functions defined by Maclaurin series, University of Michigan Studies, Scientific Series, vol. 9, 1936.

2. C. G. Fry and H. K. Hughes, Asymptotic developments of certain integral functions, Duke Math. J. vol. 9 (1942) pp. 791-802.

3. H. K. Hughes, On a theorem of Newsom, Bull. Amer. Math. Soc. vol. 49 (1943) pp. 288-292.

4. C. V. Newsom, On the character of certain entire functions in distant portions of the plane, Amer. J. Math. vol. 60 (1938) pp. 561-572.

5. C. V. Newsom, and A. Franck, Upon the asymptotic representation of functions of the Bessel type, Boletin Matematico vol. 14 (1940) pp. 11-14.

6. C. V. Newsom, The asymptotic behavior of a class of entire functions, Amer. J. Math. vol. 65 (1943) pp. 450-454.

PuRdue UnIVERsity 